\title{
How to target inter-regional phase synchronization with dual-site Transcranial Alternating Current Stimulation
}

Saturnino, Guilherme Bicalho; Madsen, Kristoffer Hougaard; Siebner, Hartwig Roman; Thielscher, Axel

Published in:

Neurolmage

Link to article, DOI:

10.1016/j.neuroimage.2017.09.024

Publication date:

2017

Document Version

Peer reviewed version

Link back to DTU Orbit

Citation $(A P A)$ :

Saturnino, G. B., Madsen, K. H., Siebner, H. R., \& Thielscher, A. (2017). How to target inter-regional phase synchronization with dual-site Transcranial Alternating Current Stimulation. Neurolmage, 163, 68-80. https://doi.org/10.1016/j.neuroimage.2017.09.024

\section{General rights}

Copyright and moral rights for the publications made accessible in the public portal are retained by the authors and/or other copyright owners and it is a condition of accessing publications that users recognise and abide by the legal requirements associated with these rights.

- Users may download and print one copy of any publication from the public portal for the purpose of private study or research.

- You may not further distribute the material or use it for any profit-making activity or commercial gain

- You may freely distribute the URL identifying the publication in the public portal 


\section{Accepted Manuscript}

How to target inter-regional phase synchronization with dual-site Transcranial Alternating Current Stimulation

Guilherme Bicalho Saturnino, Kristoffer Hougaard Madsen, Hartwig Roman Siebner, Axel Thielscher

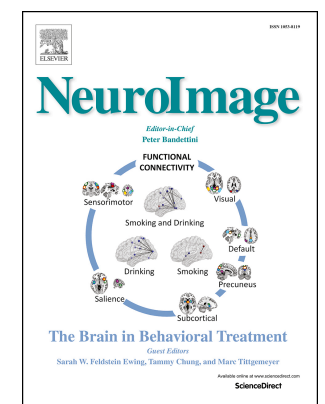

PII: S1053-8119(17)30772-3

DOI: 10.1016/j.neuroimage.2017.09.024

Reference: $\quad$ YNIMG 14337

To appear in: Neurolmage

Received Date: 29 May 2017

Accepted Date: 12 September 2017

Please cite this article as: Saturnino, G.B., Madsen, K.H., Siebner, H.R., Thielscher, A., How to target inter-regional phase synchronization with dual-site Transcranial Alternating Current Stimulation, Neurolmage (2017), doi: 10.1016/j.neuroimage.2017.09.024.

This is a PDF file of an unedited manuscript that has been accepted for publication. As a service to our customers we are providing this early version of the manuscript. The manuscript will undergo copyediting, typesetting, and review of the resulting proof before it is published in its final form. Please note that during the production process errors may be discovered which could affect the content, and all legal disclaimers that apply to the journal pertain. 


\title{
How to target inter-regional phase synchronization with
}

\section{dual-site Transcranial Alternating Current Stimulation}

\author{
Guilherme Bicalho Saturnino ${ }^{1,2}$, Kristoffer Hougaard Madsen ${ }^{1,3}$, \\ Hartwig Roman Siebner ${ }^{1,4}$, Axel Thielscher ${ }^{1,2}$
}

\section{Institutions:}

${ }^{1}$ Danish Research Centre for Magnetic Resonance, Centre for Functional and Diagnostic Imaging and Research, Copenhagen University Hospital Hvidovre, Denmark.

2 Center for Magnetic Resonance, Department of Electrical Engineering, Technical University of Denmark, Kgs Lyngby, Denmark.

3 Department of Applied Mathematics and Computer Science, Technical University of Denmark, Kgs. Lyngby, Denmark

4 Department of Neurology, Copenhagen University Hospital Bispebjerg, Copenhagen, Denmark

\section{Corresponding author}

Dr. Axel Thielscher

Danish Research Center for Magnetic Resonance

Copenhagen University Hospital Hvidovre

DK-2650 Hvidovre, Denmark

Email: axelt@drcmr.dk; Phone \#: +45 38623326 


\section{Abstract}

Large-scale synchronization of neural oscillations is a key mechanism for functional information exchange among brain areas. Dual-site Transcranial Alternating Current Stimulation (ds-TACS) has been recently introduced as non-invasive technique to manipulate the temporal phase relationship of local oscillations in two connected cortical areas. While the frequency of ds-TACS is matched, the phase of stimulation is either identical (in-phase stimulation) or opposite (anti-phase stimulation) in the two cortical target areas. In-phase stimulation is thought to synchronize the endogenous oscillations and hereby to improve behavioral performance. Conversely, anti-phase stimulation is thought to desynchronize neural oscillations in the two areas, which is expected to decrease performance. Critically, in- and anti-phase ds-TACS should only differ with respect to temporal phase, while all other stimulation parameters such as focality and stimulation intensity should be matched to enable an unambiguous interpretation of the behavioral effects. Using electric field simulations based on a realistic head geometry, we tested how well this goal has been met in studies, which have employed ds-TACS up to now. Separating the induced electrical fields in their spatial and temporal components, we investigated how the chosen electrode montages determined the spatial field distribution and the generation of phase variations in the injected electric fields. Considering the basic physical mechanisms, we derived recommendations for an optimized stimulation montage. The latter allows for a principled design of in- and anti-phase ds-TACS conditions with matched spatial distributions of the electric field. This knowledge will help cognitive neuroscientists to design optimal ds-TACS configurations, which are suited to probe unambiguously the causal contribution of phase coupling to specific cognitive processes in the human brain. 


\section{ACCEPTED MANUSCRIPT}

Keywords: Dual-site transcranial alternating current stimulation, neural oscillations,

electric field simulations, focality 


\section{Introduction}

Neural oscillations constitute an important part of the neural information processing in the human brain. In particular, the synchronization of oscillatory activity across different brain areas is thought to facilitate information transfer by temporally aligning neural processing across brain areas (Buzsaki and Draguhn, 2004; Canolty and Knight, 2010). Neural oscillations at higher frequencies that represent local fast computations are "nested" in specific phases of slower oscillations. Therefore, the commonly held notion is that synchronizing the slower oscillatory activity within functional brain networks aligns processing across brain areas (Fries, 2005). However, it is still a matter of debate how much the synchronization of neural oscillations plays a pivotal role for cognitive brain functions.

By coupling the phase of the local neuronal oscillations to that of the injected currents, transcranial alternating current stimulation (TACS) opens up unprecedented possibilities to rigorously test current concepts of the functional role of oscillatory cortical activity (Helfrich et al., 2014). This virtue of TACS has motivated several studies in which TACS was employed as non-invasive interventional tool to alter experimentally between-area synchronization of cortical oscillations in-vivo in humans (Bächinger et al., 2017; Helfrich et al., 2014; Polanía et al., 2015, 2012; Strüber et al., 2014; Violante et al., 2017). Using setups with three or more electrodes connected to one or two 2-channel stimulators, the intention was to inject selectively weak alternating currents into two brain areas simultaneously and to manipulate the phase relationship between the ongoing cortical oscillations in the two cortical target areas. So far, this dual-site TACS (ds-TACS) approach was used to contrast the effects of in- and anti-phase stimulation of two brain areas on behavioral task performance. Here, in-phase stimulation served to synchronize the endogenous oscillations by applying currents of the same temporal phase to both 
regions. Applying alternating currents of the opposite temporal phase, ds-TACS was used to desynchronize the neural oscillations of the two areas during anti-phase stimulation.

In a seminal study, Polanía et al. (2012) modulated the synchronization between frontopolar and parietal cortical areas in the theta band by means of ds-TACS which altered performance in a visual memory-matching task. A follow-up study employed a dsTACS protocol that mimicked gamma-band oscillations embedded in a theta-band envelope to modulate frontoparietal synchronization, resulting in a change of value-based choices in a decision task (Polanía et al., 2015). Two further studies targeted the interhemispheric gamma-band coherence over parieto-occipital areas by means of dsTACS, and showed that this modulated the perception of bi-stable motion patterns (Helfrich et al., 2014; Strüber et al., 2014). Together, these studies underpin the notion that synchronized oscillatory brain activity supports information transfer within functional brain networks through long-range neuronal coupling. However, the unambiguous interpretation of the behavioral results obtained with ds-TACS critically depends on the proper control of the electric field patterns created in the human brain. In order to allow for unambiguous interpretation of the changes in task performance, the in- and anti-phase stimulation conditions should only differ in the relative temporal phase of the currents applied to the two brain areas, but not in other spatial or temporal features of cortex stimulation.

To unambiguously attribute the results to a TACS-induced manipulation in the phase of cortico-cortical coupling, the in- and anti-phase ds-TACS protocols have to produce timevarying electrical current patterns that are spatially confined to the cortical target sites and the regionally injected alternating electrical fields should only differ in their phase relationship but no other electrical field properties. Yet, the choice of suited electrode montages, including the temporal waveforms of the currents injected into the single electrodes, is not straightforward. The spatial spread and temporal features of the timevarying electric field in the head volume conductor, created by the superposition of 


\section{ACCEPTED MANUSCRIPT}

currents injected into the surface electrodes can be very non-intuitive in the complex case of ds-TACS.

Here, we use electric field simulations based on a realistic head geometry to characterize the main characteristics of the electrode montages that have been used in empirical dsTACS studies so far. In particular, we test how well the goal of matched in- and anti-phase stimulation conditions has been met. We further discuss the basic physical mechanisms, which influence the generation of the phase variations in the injected electric fields. Finally, we suggest an optimized electrode montage for ds-TACS, which is better capable of targeting the same areas across in- and anti-phase conditions. Our study gives important guidance for the design of future empirical ds-TACS approaches. 


\section{Materials and method}

\section{Simulation framework}

We used the SimNIBS 2 software pipeline (Thielscher et al., 2015) for the simulations. It employs the Finite-Element method (FEM) that allows for accurate calculations of the electric field also for complex geometrical shapes such as the human head. Assuming a quasi-static regime (Miranda et al., 2014; Opitz et al., 2016), which is justified given the relatively low frequencies of ds-TACS $(<1 \mathrm{kHz})$, the fields can be separated into a spatial and a temporal component (details are stated below). We calculated the spatial component by solving Laplace's equation for the electrostatic potential $\varphi$

$$
\nabla \cdot(\sigma \nabla \varphi)=0
$$

using Dirichlet boundary conditions at the electrodes ( $\sigma$ denotes the ohmic conductivity, and $\nabla$ the divergence and gradient differential vector operators, respectively). The FEM solver (Geuzaine, 2007) used the Galerkin method based on tetrahedral first order elements, and the residuals for the conjugate gradient solver were required to be $<10^{-9}$. The electric field $\mathbf{E}$ (bold characters denote vectors) was then determined by the numerical differentiation of $\varphi$

$$
\boldsymbol{E}=-\nabla \varphi
$$

The current density $\boldsymbol{J}$ was determined via Ohm's law $\boldsymbol{J}=\sigma \boldsymbol{E}$. The electrostatic potential and the field values were scaled such that a unit current (i.e., 1) was passing through the electrodes.

The simulations were performed using a head model of a healthy volunteer (26 years, female, right-handed), that was created based on existing T1- and T2-weighted magnetic resonance images (MRI), previously collected on a 3 T TIM Trio scanner (Siemens Healthcare, Germany) equipped with a 12-channel coil at the Max Planck Institute for Biological Cybernetics (Tübingen, Germany). Details of the MRI parameters can be found in (Opitz et al., 2011). An improved version of head meshing pipeline of SimNIBS 2 was 
ACCEPTED MANUSCRIPT

used to create a tetrahedral mesh containing seven tissue types. The surface reconstruction of brain gray matter (GM) and white matter (WM) was performed using FreeSurfer 5.3.0 (Dale et al., 1999). The segmentation of skin, compact bone, spongy bone, the vitreous bodies of the eyes, the cerebrospinal fluid (CSF) and the air cavities was based on the SPM 12 toolbox (Statistical Parametric Mapping 12) implemented in Matlab (The Mathworks, Inc.), with extended tissue probability maps that covered the neck (Huang et al., 2013). Custom-written Python scripts were employed for post-hoc cleaning of the voxel segmentations, followed by the reconstruction of the tissue boundaries using triangle surface meshes. The triangle surfaces were then used to create a tetrahedral volume mesh, as further described in (Windhoff et al., 2013). The final head mesh had around 4,000,000 tetrahedra and 700,000 nodes. The conductivities of all tissues including WM were taken as isotropic, and the assigned conductivity values were $0.126 \mathrm{~S} / \mathrm{m}(\mathrm{WM})$, $0.275 \mathrm{~S} / \mathrm{m}$ (GM), $1.654 \mathrm{~S} / \mathrm{m}$ (CSF), $0.025 \mathrm{~S} / \mathrm{m}$ (spongy bone), $0.008 \mathrm{~S} / \mathrm{m}$ (compact bone), $0.50 \mathrm{~S} / \mathrm{m}$ (vitreous bodies) and $0.465 \mathrm{~S} / \mathrm{m}$ (skin) (Saturnino et al., 2015).

Unless noted otherwise, the electrode positions were chosen according to the EEG 10-20 system (Homan et al., 1987). The electrodes were modelled as a single layer of conductive gel with $1 \mathrm{~S} / \mathrm{m}$ conductivity and a thickness of $5 \mathrm{~mm}$. The electrical potential was constant for the entire electrode surface, which is equivalent to using a very well conductive electrode pad on top of the gel (Saturnino et al., 2015). We did not use a more detailed modeling of the electrodes, e.g. including the connector positions, as assessing the effects of different electrode materials was not in the focus of this study.

\section{Linearity and superposition of electric fields}

In the case of two channels (one "active" channel and one "return" channel), the electric field created at position $\boldsymbol{p}$ in the head and at time point $t$ is determined by the product of 
ACCEPTED MANUSCRIPT

the spatial component (eq. 2) and the time course of the current injected into the active channel:

$$
\boldsymbol{E}(\boldsymbol{p}, t)=\boldsymbol{E}(\boldsymbol{p}) I(t)
$$

This linear dependence results from the assumptions of a quasi-static regime and a purely ohmic volume conductor. For the general case of $n$ active channels with injected currents $I_{i}(t), i=1,2, \ldots n$, and one return electrode with the resulting current $I_{r}=-\sum_{i} I_{i}$, the electric field $\boldsymbol{E}(\boldsymbol{p}, t)$ is given by the linear superposition of the $\mathrm{n}$ electric fields $\boldsymbol{E}_{i}(\boldsymbol{p}, t)$ :

$$
\boldsymbol{E}(\boldsymbol{p}, t)=\sum_{i=1}^{N} \boldsymbol{E}_{i}(\boldsymbol{p}, t)=\sum_{i=1}^{N} \boldsymbol{E}_{i}(\boldsymbol{p}) I_{i}(t)
$$

This is similar to the concept of "lead fields" used in EEG (Dmochowski et al., 2011). Equation 4 allows for a time-efficient calculation of the injected fields in practice and simplifies the theoretical analyses of the fields resulting from the different montages.

\section{Electrode Montages and Current Profiles}

\section{Electrode Montage 1}

The first montage (Fig. 1A) mimicked the protocol described in (Polanía et al., 2012), and used active electrodes over F3 and over P3, each connected to a different channel, and a return electrode over Cz. All three electrodes were circular electrodes with $30 \mathrm{~mm}$ diameter. The currents through each electrode (Fig. 1B) are given by

$$
\begin{gathered}
I_{F 3}(t)=I_{0} \sin (2 \pi f t) \\
I_{P 3}(t)=I_{0} \sin (2 \pi f t+\phi) \\
I_{R}(t)=-\left(I_{F 3}(t)+I_{P 3}(t)\right)
\end{gathered}
$$

whereby the phase difference $\phi$ between both currents was $\phi=0$ for the in-phase condition, and $\phi=\pi$ (i.e., 1809 for the anti-phase condition. A stimu lation frequency of $f=6$ $\mathrm{Hz}$ was used in the main experimental condition and a peak current amplitude of $\mathrm{I}_{0}=0.5$ $\mathrm{mA}$ (corresponding to $1 \mathrm{~mA}$ peak to peak) was applied. It can be easily seen from the 
above equation that the two currents injected in the return electrode add up for the inphase condition, resulting a peak current amplitude of $I_{R_{-} P e a k}=2 I_{0}=1 \mathrm{~mA}$. In contrast, they fully cancel out for the anti-phase condition, so that $I_{R}=0$ at all times (using the fact that $\sin (x+\pi)=-\sin (x))$. Using equations 4 and 5 , the total electric field at position $\boldsymbol{p}$ in the brain and time $t$ then is

$$
\boldsymbol{E}(\boldsymbol{p}, t)=I_{0}\left(\boldsymbol{E}_{F 3}(\boldsymbol{p}) \pm \boldsymbol{E}_{P 3}(\boldsymbol{p})\right) \sin (2 \pi f t)
$$

for the in-phase $(+)$ and anti-phase (-) conditions. Notably, we can separate the total electric field into a spatial and temporal component

$$
\boldsymbol{E}(\boldsymbol{p}, t)=\boldsymbol{P}(\boldsymbol{p}) T(t)
$$

The spatial component $\boldsymbol{P}(\boldsymbol{p})=I_{0}\left(\boldsymbol{E}_{F 3}(\boldsymbol{p}) \pm \boldsymbol{E}_{P 3}(\boldsymbol{p})\right)$ depends only in the position $\boldsymbol{p}$ in the cortex, and $T(t)=\sin (2 \pi f t)$ depends only on the time point $t$. Therefore, for montage 1 , all positions in the brain will be stimulated with the same waveform having either the same or the opposite phase (as the temporal component stays the same, but the spatial component can have opposite directions relative to the local orientation of the cortical sheet).

\section{Electrode Montage 2}

The second montage (Fig. 2A) replicated the protocol described in (Polanía et al., 2015), which employed three electrodes. The two active electrodes had rectangular shapes, with sizes of $70 \times 50 \mathrm{~mm}^{2}$, and were placed over $\mathrm{Fpz}$ and $\mathrm{Pz}$ and each was connected to a different channel. A rectangular $60 \times 60 \mathrm{~mm}^{2}$ return electrode was placed on the right lower part of the neck to mimic the placement of the reference electrode on the right shoulder used in (Polanía et al., 2015) as closely as possible. Given the distance of the two positions to the brain, we do not expect that this difference will change our findings qualitatively. Here, we use the term "return electrode" to denote electrodes that are intended not to contribute to the physiological effects, and correspondingly "return 
channel" to indicate the channels connected to these electrodes. The montage used an oscillation with $f_{1}=55 \mathrm{~Hz}$ which is modulated by an envelope with a frequency of $f_{2}=$ $6 \mathrm{~Hz}$. In the in-phase condition, both oscillations were applied at the same phase at both active electrodes, while both oscillations were shifted by $180^{\circ}$ in the anti-phase condition. The current through each electrode (Fig. 2B) is thus given by

$$
\begin{gathered}
I_{F p Z}=I_{0}\left(1-\cos \left(2 \pi f_{2} t\right)\right) \sin \left(2 \pi f_{1} t\right) \\
I_{P Z}=I_{0}\left(1-\cos \left(2 \pi f_{2} t+\phi\right)\right) \sin \left(2 \pi f_{1} t+\phi\right) \\
I_{R}=-\left(I_{F p Z}+I_{P Z}\right)
\end{gathered}
$$

Again, the phase difference is $\phi=0$ in the in-phase condition, and $\phi=\pi$ in the anti-phase condition. A peak current amplitude of $I_{0}=1 \mathrm{~mA}$ (corresponding to $2 \mathrm{~mA}$ peak to peak) was used. In the in-phase condition, the currents add up at the return electrode, with a peak current amplitude of $I_{\text {R_Peak }}=2 I_{0}=2 \mathrm{~mA}$. We can again separate the total electric field using equation 7 into the spatial component $\boldsymbol{P}(\boldsymbol{p})=I_{0}\left(\boldsymbol{E}_{m F P C}(\boldsymbol{p})+\boldsymbol{E}_{P C}(\boldsymbol{p})\right)$ and the temporal component $T(t)=\left(1-\cos \left(2 \pi f_{2} t\right)\right) \sin \left(2 \pi f_{1} t\right)$. That is, all positions in the brain will be stimulated with the same waveform during the in-phase condition. However, in the anti-phase condition, the current waveform injected into $\mathrm{Pz}$ is not simply the negative of the waveform injected into $\mathrm{Fpz}$, so that the equation for the total field $\boldsymbol{E}(\boldsymbol{p}, t)$ is not separable anymore. The important consequence of this is that the resulting temporal waveform in the brain will change with position, i.e. it is a spatially varying mixture of the two waveforms injected into the active electrodes.

\section{Electrode Montage 3}

The third montage (Fig. 3A) replicated the setting described in (Strüber et al., 2014). It used a 2-channel stimulator with sinusoidal currents, and the currents in the two channels (Fig. 3B) are given by 


$$
\begin{gathered}
\text { ACCEPTED MANUSCRIPT } \\
\begin{array}{c}
I_{1}(t)=I_{0} \sin (2 \pi f t) \\
I_{R}(t)=-I_{1}(t)
\end{array}
\end{gathered}
$$

In this case, the total electric field in the brain is always separable (equation 7) as

$$
\boldsymbol{E}(\boldsymbol{p}, t)=I_{0} \boldsymbol{E}(\boldsymbol{p}) \sin (2 \pi f t)
$$

and all positions in the brain experience the same temporal waveform. In- and anti-phase conditions were created by changing the number and spatial configuration of the electrodes to alter the spatial component $\boldsymbol{E}(\boldsymbol{p})$ of the field. In the in-phase condition, four rectangular 39×39 $\mathrm{mm}^{2}$ electrodes were placed above positions $\mathrm{C} 3, \mathrm{C} 4, \mathrm{O} 1$ and $\mathrm{O} 2$. The electrodes above $\mathrm{C} 3$ and $\mathrm{C} 4$ were commonly connected to the first channel, and the electrodes above $\mathrm{O} 1$ and $\mathrm{O} 2$ commonly to the second channel. By that, it was ensured that the currents injected into the two occipital electrodes (O1 and O2) were in-phase. The stimulation frequency of the main condition simulated here was $40 \mathrm{~Hz}$, and the intensity was individually titrated in (Strüber et al., 2014), with an average peak current amplitude of $\mathrm{I}_{0}=0.615 \mathrm{~mA}$ (corresponding to $1.23 \mathrm{~mA}$ peak to peak amplitude). In the anti-phase condition, two rectangular $50 \times 70 \mathrm{~mm}^{2}$ electrodes were placed between positions P7-PO7 and between P8-PO8, and the average peak amplitude was $0.51 \mathrm{~mA}$, corresponding to $1.02 \mathrm{~mA}$ peak to peak amplitude.

\section{Electrode Montage 4}

The fourth montage related to the montage used in (Helfrich et al., 2014), which employed two $4 \times 1$ montages centered above left and right parietal-occipital regions (Fig. 4A). The single electrodes had a diameter of $12 \mathrm{~mm}$. A 2-channel stimulator was used, so that the above equation 9 also applies for this electrode montage. The in- and anti-phase conditions were created by changing the connection pattern between the stimulator and the electrodes. In the in-phase condition, both central electrodes were connected to the first channel, while all peripheral electrodes were connected to the second channel. In the 


\section{ACCEPTED MANUSCRIPT}

anti-phase condition, all the electrodes over the right hemisphere were connected to the first channel and all the electrodes over the left hemisphere to the second channel. The stimulation frequency was $40 \mathrm{~Hz}$, and the peak amplitude was $I_{0}=0.5 \mathrm{~mA}(1 \mathrm{~mA}$ peak to peak).

\section{Ring montages}

In addition to the above montages that were motivated by existing experimental studies, we explored the combination of two center-surround ring montages. Each montage consisted of a small circular center electrode with a diameter of $20 \mathrm{~mm}$, and a surrounding ring electrode with inner and outer diameters of $40 \mathrm{~mm}$ and $60 \mathrm{~mm}$. We tested a situation in which the two montages were placed symmetrically above each hemisphere (Fig. 5A), and the case that they were placed closely to each other while preventing a direct contact between the rings (Fig. 6A). We denote the inner circular electrodes as $\mathrm{C} 1$ and $\mathrm{C} 2$, and the outer ring electrodes as R1 and R2. The currents used to drive the electrodes (Fig. 5B) are

$$
\begin{gathered}
I_{C 1}=I_{0} \sin (2 \pi f t) \\
I_{R 1}=-I_{C 1} \\
I_{C 2}=I_{0} \sin (2 \pi f t+\phi) \\
I_{R 2}=-\left(I_{C 1}+I_{R 1}+I_{C 2}\right)=-I_{C 2}
\end{gathered}
$$

For the in- and anti-phase conditions, the total electric field $E(p, t)$ can be separated into spatial and temporal components and written as:

$$
\boldsymbol{E}(\boldsymbol{p}, t)=I_{0}\left(\boldsymbol{E}_{C 1}(p)-\boldsymbol{E}_{R 1}(p) \pm \boldsymbol{E}_{C 2}(p)\right) \sin (2 \pi f t)
$$

Here, we arbitrarily chose ring R2 as "return" electrode when calculating the static field components (the choice does not affect the resulting electric field). The peak amplitude was set to $\mathrm{I}_{0}=0.5 \mathrm{~mA}$ ( $1 \mathrm{~mA}$ peak to peak), and the stimulation frequency to $40 \mathrm{~Hz}$. In additional simulations, we tested the possibility to adjust the stimulation currents of the two 


\section{ACCEPTED MANUSCRIPT}

ring montages independently from each other in order to achieve the same electric field strength in the two target areas (modelled by setting the peak current amplitude of $I_{C 2}$ to $c \cdot \mathrm{I}_{0}$, with constant $\mathrm{c}=1.5$ empirically determined from the first simulation results).

We explored the possibility of driving both ring montages with a single 2-channel stimulator (equation 9 applies in this case) (Fig. 5D-F). For the in-phase condition, C1 and C2 are connected to the active channel, and R1 and R2 to the return channel. In the anti-phase condition, C1 and R2 are connected to the active channel, while C2 and R1 are connected to the return channel. Given that now both montages were driven by a single source, we set $I_{0}=1.0 \mathrm{~mA}$ ( $2 \mathrm{~mA}$ peak to peak) to keep the total amount of injected current identical to the upper case.

Additionally, we explored the effect of ring size and inter-electrode distance (Fig. 7). We placed one center-surround ring montage close to the C5 position, and another one 65 $\mathrm{mm}$ superior to it. We then systematically varied the second montage position on an arch across scalp surface going though $\mathrm{C} 5-\mathrm{Cz}-\mathrm{C} 3$ in $5 \mathrm{~mm}$ steps. We also varied the ring diameter from $60 \mathrm{~mm}$ to $100 \mathrm{~mm}$ in $10 \mathrm{~mm}$ steps, while keeping the inner electrode diameter and the width of the ring $\left(\emptyset_{\text {external }}-\emptyset_{\text {internal }}\right)$ constant (both at $20 \mathrm{~mm}$ ). Simulations were only performed if the two rings had a minimal distance of $5 \mathrm{~mm}$ to each other.

\section{Data Extraction and Visualization}

Given the large differences between the stimulation approaches, we focused mostly on a qualitative description to point out their specific advantages and disadvantages. The goals of the in- and anti-phase conditions of ds-TACS experiments were to create either temporally synchronous or anti-synchronous polarizations of the cortical sheet in the target brain areas. This is thought to modulate rhythmically the polarization of the pyramidal cells 
ACCEPTED MANUSCRIPT

either in phase between the two target areas or at opposite phase. This polarization is driven by the normal component of the electric field, i.e. the component that is orthogonal to the local orientation of the cortex. Therefore, we extracted the normal component of the field in the middle of gray matter using custom-written software (Bungert et al., 2016) for visualization. Electric fields flowing from CSF into gray matter, and therefore depolarizing the somas of pyramidal cells, were given positive values (corresponding to anodal stimulation). Negative values indicate cathodal stimulation. However, it is well possible that also the electric field components that are tangential to the gray matter sheet influence neural activity (Rahman et al., 2013), albeit in a non-predictable way. Therefore, we also visualized the electric field vectors in the middle of the gray matter sheet in selected cases in order to point out brain areas that experienced high field intensities.

\section{Metrics}

In order to quantitatively characterize the electric field distributions, we computed four metrics for the normal component of the electric field that was read out in the middle of the gray matter sheet:

1. Maximum of the absolute value of the normal component $\max \left(\left|\boldsymbol{E}_{n}\right|\right)$ :

Calculated as the $99.9^{\text {th }}$ percentile of the absolute value of the normal component over the entire gray matter sheet.

2. Stimulated Area:

Fraction (in \%) of the gray matter sheet in which the absolute value of the normal component exceeds $80 \%$ of the maximum absolute value. The smaller the value of this metric, the more focal the field. The choice of $80 \%$ was arbitrary, and other values in this range give similar results.

3. Relative Distance Measure (RDM): 
In order to measure the similarity of the spatial field distributions between the inand anti-phase conditions, we calculated the relative distance measure (RDM) (Meijs et al., 1989) of the absolute values of normal components in the in- and antiphase conditions

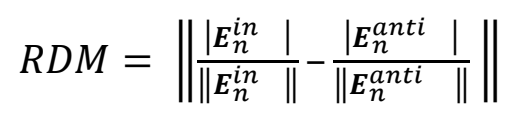

where $\boldsymbol{E}_{n}^{i n}$ and $\boldsymbol{E}_{n}^{a n t i}$ are vectors containing the electric field normal to the middle of gray matter sheet in the in- and anti-phase conditions respectively. $\|\cdot\|$ is the Euclidian norm. As both numbers are positive in our case, RDM varies between 0 (identical field distributions) and $\sqrt{2}$ (highly dissimilar distributions).

4. Normalized Dot Product (DotP) :

The normalized dot product

$$
\operatorname{DotP}=\frac{\boldsymbol{E}_{n}^{i n} \cdot \boldsymbol{E}_{n}^{\text {anti }}}{\left\|\boldsymbol{E}_{n}^{i n}\right\|\left\|\boldsymbol{E}_{n}^{\text {anti }}\right\|}
$$

was used to access the orthogonality of the two electric field distribution (where $\boldsymbol{E}_{n}^{i n} \cdot \boldsymbol{E}_{n}^{a n t i}=\sum_{\boldsymbol{i}=\mathbf{0}}^{N} E_{n}^{i n, i} E_{n}^{a n t i, i}$ ). The normalized dot product varies between -1 (fields are proportional, but of opposite sign) and 1 (fields are proportional and of the same magnitude). A value of 0 indicates that the fields are orthogonal.

In the ideal case, the in- and anti-phase conditions have similar maximal field strengths $\max \left(\left|\boldsymbol{E}_{n}\right|\right)$ and similar focalities. In addition, they have a RDM close to 0 (meaning that they stimulate the same areas) and a DotP close to 0 (meaning that the anti-phase condition affects half of the stimulated areas with opposite phase).

\section{Results}

Electrode Montage 1 


\section{ACCEPTED MANUSCRIPT}

The different involvement of the return electrode in the in- and antiphase conditions (Fig. 1C\&D) has a strong effect on the resulting field patterns. During in-phase stimulation, the cortex area underneath the return electrode is most strongly polarized by the alternating currents (left column of Fig. 1C), while the polarization directly underneath the active electrodes is weaker. This results from the fact that the current strength in the return electrode is twice the current strength in each of the active electrodes. In contrast, the strongest polarizations are shifted towards positions close to the active electrodes for the anti-phase stimulation (right column of Fig. 1C). The large RDM shows that the spatial

distribution of the field does indeed change between conditions ( $1^{\text {st }}$ row of Table 1$)$. Strong tangential electric fields occur in the gyral crowns situated between the electrodes (Fig. 1D) for both conditions, and the field directions change clearly between the two conditions.

\section{Electrode Montage 2}

During in-phase stimulation (left column of Fig. 2C), the currents from both electrodes 1 and 2 flow to the return electrode, which results a very strong co-stimulation of orbitofrontal, dorsal and ventral occipital and temporal areas and the cerebellum. Many of these areas experience anti-phasic stimulation. Similar areas are also co-stimulated during the anti-phase condition (middle and right columns of Fig. 2C), albeit to a weaker extent. This is because the currents injected into the active electrodes do not peak at the same time point anymore. In addition, the currents flow not only from the active electrodes to the return electrode, but also directly between the active electrodes.

The time courses of the normal component of the electric field at selected cortical positions are shown in Figure 2D. It can be seen that the montage generates the intended time courses in the regions underneath the active electrodes both for the in- and anti-phase conditions (rows 1 and 2 in Fig. 2D), closely following the time courses injected into the electrodes (Fig. 2B). The co-stimulated areas have the same time course for the in-phase 


\section{ACCEPTED MANUSCRIPT}

condition. However, in the anti-phase condition, their time courses are mixtures of the current waveforms injected into the electrodes. For example, the $55 \mathrm{~Hz}$ oscillation is modulated by envelope having twice the original frequency of $6 \mathrm{~Hz}$ in the cerebellum (right part of row 3). The high RDMs for the time points in the middle and at the end of the oscillation show that the distribution of the electric field differs significantly from the field in the in-phase condition ( $2^{\text {nd }}$ and $3^{\text {rd }}$ row of Table 1). The DotP of 0,82 and $-0,84$ shows that the fields at these two time points are far from the ideal situation in which half of the areas would be stimulated with the opposite phase during the anti-phase compared to the inphase conditions (which would be indicated by a DotP of 0 ).

\section{Electrode Montage 3}

The field patterns differ very clearly between the two electrode montages (Fig. 3A) used for in- and anti-phase stimulation $\left(4^{\text {th }}\right.$ column of Table 1$)$. In the in-phase condition (first column of Fig. 3C), strong fields occur bilaterally in the posterior occipital cortices, and in addition bilaterally in posterior parietal and temporal areas. As intended, the fields in the occipital cortices are in phase between both hemispheres. The fields in posterior parietal and temporal areas tend to have the opposite phase to the occipital areas. However, at many gyri, the pattern alternates between in- and anti-phase depending on the side of the gyrus.

The anti-phase stimulation (second column of Fig. $3 \mathrm{C}$ ) affects mostly parietal-occipital areas and the cerebellum. The peak fields occur more laterally compared to those seen for the in-phase condition. As intended, the stimulation of the parietal-occipital areas of the two hemispheres occurs at opposite phase. However, also the stimulation of the lateral and medial occipital areas of the same hemisphere occurs at opposite phase (inserts in Fig. 3C). 


\section{Electrode Montage 4}

This montage achieves focal electric fields bilaterally in lateral occipital areas for the inphase condition (first column of Fig. 4C). In the anti-phase condition (second column of Fig. 4C), however, the fields are more unfocal and about two times stronger and affect occipital and posterior temporal and parietal areas ( $5^{\text {th }}$ row of Table 1$)$. Similar to the antiphase condition in Fig. 3C, the lateral occipital areas of the two hemispheres are stimulated with the intended opposite phases, but also the medial occipital areas are strongly stimulated at opposite phase to the lateral areas of the same hemisphere (inserts in Fig. 4C).

\section{Ring Montages}

The combination of two center-surround ring montages (Fig. 5) achieves very similar spatial distributions during the in- and anti-phase conditions and at good orthogonality between both conditions ( $6^{\text {th }}$ row in Table 1), with only the relative temporal phases of the two target areas changing between both stimulation conditions. The currents are flowing from the circular electrodes to the surrounding rings, and therefore do not influence each other. As shown in Fig. 5D-F, if a 2-channel stimulator is used instead of a 4-channel stimulator (or two synchronized 2-channel stimulators), we observe a large current flow between the 2 ring electrodes in the anti-phase condition. This results in the undesired stimulation of large parts of the cortex in the anti-phase condition (stimulated area for 4 channel montage is $0,27 \%$; stimulated area for 2 channel montage is $0,82 \%$ ) .

As this approach uses the two rings to "shield" the center electrodes from each other, it requires some distance between both rings to work properly. When both rings are moved close to each other (Fig. 6), direct current flow between the two central electrodes occurs for the anti-phase condition (right column of Fig. 6D). This results in a co-stimulation of the 


\section{ACCEPTED MANUSCRIPT}

brain region between the two pairs, with the strongest effects occurring at the gyral crowns by currents being tangential to the cortical sheet and significantly reduces the similarity between field distributions across the in- and anti-phase conditions ( $R D M=0,56)$. An advantage of the ring approach is a localized control of the electric field strength for each target area separately (Fig. 6E). As example, the current flowing in the posterior electrodes has been adjusted in order to obtain the same value for the normal component of the electric field in both target regions.

Fig. 7 shows a clear increase in $\max \left(\left|\boldsymbol{E}_{n}\right|\right)$ with the ring diameter, accompanied by more unfocal fields. We can also see that, as the two rings approach each other, the RDM becomes significantly worse, indicating that the in- and anti-phase conditions start to stimulate different cortical regions. DotP appears to have a strong dependency on the position of the second electrode. It maintains acceptable levels for distances between 155 $\mathrm{mm}$ and $125 \mathrm{~mm}$, but gets increasingly worse when the distance is decreased further. For distances of $85 \mathrm{~mm}$ and below, the orthogonality is increasing again. However, this is simply due to the fields displaying less overlapping in general, as indicated by the increasing RDM at those distances. Taken together, these results indicate that the simulated two ring approaches work reasonably well for distances of $3 \mathrm{~cm}$ or more between the outer ring diameters. For even closer distances, individualized simulations are recommendable.

\section{Discussion}

Electrode montages for ds-TACS should ideally allow for in- and anti-phase stimulation conditions, which differ only in the temporal phase of the electric fields injected in the target brain areas, but are matched for all other stimulation features such as peak field strengths and focality. Our analysis based on detailed simulations of the electric fields in a 
realistic head model indicate that this goal was not reached in the four ds-TACS setups taken from existing studies (Helfrich et al., 2014; Polanía et al., 2015, 2012; Strüber et al., 2014) (Fig. 1-4). In particular, additional areas besides the target regions were costimulated, and the spatial pattern of this effect differed between the in- and anti-phase conditions. Our results raise the possibility that these differences in the temporo-spatial properties between the used in-phase and anti-phase ds-TACS protocols might have contributed to the experimentally measured effects on behavior, challenging a straightforward attribution of the behavioral results to a phase manipulation of intrinsic neural activity in the targeted brain regions. For instance, if the co-stimulated areas in a ds-TACS experiment are part of the same functional network as the main target areas and as tested by the behavioral task, then this may clearly change the results, while the impact may be negligible otherwise.

The main point of this study is that putative ambiguities can and should be ruled out in the first instance by selecting electrode montages for which in- and anti-phase ds-TACS effectively induces the intended difference in the temporal phase of the electric fields injected in the target brain areas, but otherwise are matched for the other electrical field properties. As example, we chose the combination of two center-surround ring montages (Fig. 5). These montages allow for a finer control of the spatial peak fields than standard approaches based on large rectangular electrode pads (Bortoletto et al., 2016). They reliably generate radially directed electric fields in the gyral crowns underneath the center electrodes, which should efficiently polarize these parts of the cortex. Compared to the more non-focal stimulation by standard approaches, this comes at the cost of more superficial brain stimulation. It should be noted that the symmetry of the electric fields generated by ring montages depends on the ohmic conductivity of the rubber rings and of the electrode gel, which should be chosen high enough (at least $10 \mathrm{~S} / \mathrm{m}$ or higher for the rubber rings; (Saturnino et al., 2015). Here, we set the electrode surfaces to spatially 
constant potentials, assuming a highly conductive electrode (e.g. based on a metal-mesh).

The radii of the outer rings influence the achievable peak electric field strengths in the cortex, with smaller rings resulting in more focal but weaker stimulation (Fig. 7). This is very similar to cross-like $4 \times 1$ montages with four peripheral and one central electrode of opposing polarity, as used by Helfrich et al. in the in-phase TACS condition (Helfrich et al., 2014), for which the relation between montage size and the field in the brain was systematically explored in (Alam et al., 2016). It can be argued that $4 \times 1$ montages can represent an improvement over ring montages if the impedance of each surrounding electrode is individually controlled. In contrast, the impedance over a ring electrode might vary, which is difficult to control. A similar effect can occur for a $4 \times 1$ montage when all surrounding electrodes are connected to a common return channel, potentially resulting in different current strengths in each of the surrounding electrodes. However, while $4 \times 1$ montages with individually controlled channels might result in a more balanced stimulation, their practical application can represent a challenge for ds-TACS, since a stimulator with a high number of channels (10) or many synchronized stimulators are required.

When the two ring montages are placed too close together, the coupling between them due to volume conduction of the head begins to be relevant, influencing in particular the anti-phase condition (Fig. 6). In that case, a marked direct current flow between the two center electrodes results in a potential co-stimulation of the brain areas between the two rings, as well as a strong current shunting through the skin at the positions where the rings are close to each other. It might be useful to explore targeting approaches based on multielectrode optimization (Dmochowski et al., 2011) to achieve better results in these cases. We showed that the in-phase simulation condition can be achieved by connecting both ring montages to a single 2-channel stimulator, as long as a mono-frequency sinusoidal waveform is used. However, this approach does not give control over the current flow through each electrode individually. For the anti-phase condition, this has undesired and 


\section{ACCEPTED MANUSCRIPT}

unexpected consequences, as shown in Fig. $5 \mathrm{~F}$. The current flow follows the pathways of least resistance, which are in this case mainly through the skin from the central to the ring electrodes, as well as through CSF and brain tissue between the ring electrodes. Very little current flows from the central electrodes through the brain into the rings.

An advantage of combining a 4-channel stimulator with realistic individualized field calculations is that the current flow can be adjusted to account for individual anatomical features (Fig. 6). By increasing the current flow in the posterior ring montage, the differences in the field strengths, mainly due to locally different skull thicknesses, could be corrected. Given the good spatial separation of the electric field patterns generated by the two ring montages, they are also well suited for the controlled application of more complex temporal waveforms using a multi-channel approach as done in (Polanía et al., 2015). For arbitrary waveforms, the electric fields injected by the two ring montages will not be mathematically separable anymore. That is, the time courses of the electric fields can be mixtures of the current waveforms injected into the electrodes. However, this will mainly occur at positions, which are not in the foci of the montages and where the absolute field strength is low.

Taken together, we have shown that the electrode montages for ds-TACS should be carefully chosen in order to prevent unwanted co-stimulations of non-target areas and to allow for an unambiguous interpretation of the experimentally observed behavioral and electrophysiological stimulation effects. We suggest the combination of two focal centersurround ring or $4 \times 1$ montages driven by a multi-channel stimulator (or two synchronized 2-channel stimulators) for phase-targeting with ds-TACS in order to ensure that the desired field patterns are created for both the in- and anti-phase conditions. 


\section{Acknowledgements}

This work was supported by a project grant sponsored by Lundbeckfonden (PI: Axel Thielscher; Grant No. R118-A11308), a Grant of Excellence "ContAct” sponsored by Lundbeckfonden (PI: Hartwig Siebner; Grant No. R59 A5399) and an Interdisciplinary Synergy Grant "Basics" sponsored by NovoNordisk fonden (recipients: Hartwig Siebner, Axel Thielscher \& Lars K Hansen, Grant No. NNF14OC0011413). 


\section{Figure legends}

Figure 1: Electrode Montage 1 (Polanía et al., 2012). A) Head model and Electrode placements on the scalp. B) Current flow through electrode 1 (anterior), electrode 2 (posterior) and the return electrode (central) for the in- and anti-phase conditions. The red vertical lines mark the time points at which the electric field distributions are shown in other subplots. The black vertical bar indicates $2 \mathrm{~mA}$, and the horizontal bar $50 \mathrm{~ms}$. C) Normal component of the electric field at the selected time point for in-phase (left) and anti-phase (right) stimulation. Positive values indicate anodal stimulation, negative values indicate cathodal stimulation. The field at other time points is proportional (with scaling factors ranging from -1 to 1 ) to the depicted distributions. The electrode outlines are indicated by the black circles. D) Electric field vectors for the same time points (left: in-phase; right: anti-phase).

Figure 2: Electrode Montage 2 (Polanía et al., 2015) A) Electrode placements on the scalp. B) Current flow through electrode 1 (anterior), electrode 2 (posterior) and the return electrode (right lower neck) for the in- and anti-phase conditions. The vertical lines mark the time points at which the electric field distributions are shown in other subplots. C) Normal component of the electrical field in the in- and anti-phase stimulation conditions. The left column shows the time point during the in-phase stimulation when the current flow through the electrodes is maximal. The middle column shows the time point in the antiphase condition where the current flow though the frontal electrode in maximal, and the right column when the current flow though the parietal electrode is maximal. D) Time courses of the normal component of the electric field at selected cortical positions. 
Figure 3: Electrode montage 3 (Strüber et al., 2014). A) Electrode placements on the scalp for in-phase stimulation (first two images) and for anti-phase simulation (last two images). B) The current flow through channel 1 and the return channel is identical for the in- and anti-phase conditions. The phase differences in the target brain areas are created by the different electrode montages. C) Normal component at one of the two time points during in- and anti-phase stimulation when the current flow through the electrodes is strongest. The insets show the medial occipital regions during anti-phase stimulation, which are stimulated at opposite phase to the parietal-occipital regions of the same hemisphere.

Figure 4: Electrode montage 4 (Helfrich et al., 2014). A) Electrode placements on the scalp. B) The current flow through channel 1 and the return channel is the same in the inand anti-phase conditions. The phase differences are created by changing the wiring of the electrodes to the channels. In the phase condition, the two center electrodes of each $4 \times 1$ montage are connected to channel 1 , while the peripheral are connected to channel 2 . In the antiphase condition, the five electrodes over the right hemisphere are connected to channel 1, while the five electrodes over the left hemisphere are connected to channel 2. C) Normal component at one of the time points during in- and anti-phase stimulation when the current flow through the electrodes is strongest. The insets show the medial occipital regions during anti-phase stimulation, which are stimulated at opposite phase to the parietal-occipital regions of the same hemisphere (similar to the anti-phase condition for electrode montage 3). 


\section{ACCEPTED MANUSCRIPT}

Figure 5: Ring montages placed symmetrically over both hemispheres. The anatomical target areas were arbitrarily selected. A) Electrode placements on the scalp. B) Current flow through center 1 , ring 1 , center 2 and ring 2 for the in- and anti-phase conditions. C) Normal component at one of the two time points during in- and anti-phase stimulation when the current flow through the electrodes is strongest. D) Montage using a 2-channel stimulator. In the in-phase condition, both central electrodes are connected to channel 1 , and both ring electrodes to channel 2 . In the anti-phase condition, the central electrode over the left hemisphere and the ring electrode over the right hemisphere are connected to channel 1, and the remaining central and ring electrodes to channel 2. E) Current flow through each channel when using a 2-channel stimulator. F) Normal component for in- and anti-phase stimulation using 2 channels.

Figure 6: Ring montages placed closed to each other. A) Electrode placements on the scalp. B) Current flow through electrode 1, ring 1, electrode 2 and ring 2 for the in- and anti-phase conditions. C) Normal component at one of the time points during in- and antiphase (left) and anti-phase stimulation (right) when the current flow through the electrodes is strongest. D) Electric field vectors for the same time points E) Normal component for the same time points, when increasing the current flow though the posterior ring montage by a factor of 1.5 .

Figure 7: $\max \left(\left|\boldsymbol{E}_{n}\right|\right)$, stimulated area, RDM and DotP for rings of different diameters and at different distances. The diameter of the center electrode and the width of the disk were kept constant while the diameter of the ring varied. One electrode was fixed at a position close to C5, while the other one was moved in $5 \mathrm{~mm}$ steps along an arch reaching over C3 and $\mathrm{Cz}$ to $\mathrm{C5}$. Notice that the "Distance" axis does not refer to the Euclidian distance, but to the distance along the arch. 


\begin{tabular}{|c|c|c|c|c|c|c|}
\hline \multicolumn{7}{|c|}{ ACCEPTED MANUSCRIPT } \\
\hline Montage & $\begin{array}{l}\max \left(\left|\boldsymbol{E}_{n}\right|\right) \\
\text { (In-Phase) } \\
\mathrm{V} / \mathrm{m}\end{array}$ & $\begin{array}{l}\max \left(\left|\boldsymbol{E}_{n}\right|\right) \\
\text { (Anti- } \\
\text { phase) V/m }\end{array}$ & $\begin{array}{l}\text { Stimulated } \\
\text { Area (In- } \\
\text { Phase) [\%] }\end{array}$ & $\begin{array}{l}\text { Stimulated } \\
\text { Area (Anti- } \\
\text { Phase) [\%] }\end{array}$ & RDM & DotP \\
\hline $\begin{array}{l}\text { Electrode } \\
\text { Montage } 1 \\
\text { (Fig. 1) }\end{array}$ & 0,183 & 0,206 & 0,49 & 0,44 & 0,83 & $-0,25$ \\
\hline $\begin{array}{l}\text { Electrode } \\
\text { Montage 2, } \mathrm{t}= \\
77.3 \mathrm{~ms} \\
\text { (Fig. 2) }\end{array}$ & 0,287 & 0,196 & 1,15 & 0,39 & 0,53 & 0,82 \\
\hline $\begin{array}{l}\text { Electrode } \\
\text { Montage 2, } \mathrm{t}= \\
159.1 \mathrm{~ms} \\
\text { (Fig. 2) }\end{array}$ & 0,005 & 0,185 & 1,15 & 0,86 & 0,50 & $-0,84$ \\
\hline $\begin{array}{l}\text { Electrode } \\
\text { montage } 3 \\
\text { (Fig. 3) }\end{array}$ & 0,202 & 0,168 & 0,73 & 0,72 & 0,84 & $-0,01$ \\
\hline $\begin{array}{l}\text { Electrode } \\
\text { montage } 4 \\
\text { (Fig. 4) }\end{array}$ & 0,072 & 0,157 & 0,21 & 0,60 & 0,87 & 0,02 \\
\hline $\begin{array}{l}\text { Ring montages } \\
(4 \mathrm{ch}) \\
\text { (Fig. 5A-C) }\end{array}$ & 0,078 & 0,074 & 0,26 & 0,27 & 0,25 & $-0,14$ \\
\hline $\begin{array}{l}\text { Ring montages } \\
\text { ( } 2 \mathrm{ch}) \\
\text { (Fig. 5D-F) }\end{array}$ & 0,038 & 0,053 & 0,27 & 0,82 & 1,04 & $-0,05$ \\
\hline $\begin{array}{l}\text { Ring montages } \\
\text { (close by) } \\
\text { (Fig. 6A-D) }\end{array}$ & 0,053 & 0,067 & 0,17 & 0,22 & 0,56 & 0,23 \\
\hline $\begin{array}{l}\text { Ring montages } \\
\text { (close by, indiv. } \\
\text { adjusted) } \\
\text { (Fig 6E) }\end{array}$ & 0,058 & 0,075 & 0,21 & 0,30 & 0,58 & $-0,20$ \\
\hline
\end{tabular}

Table 1: Metrics for each montage simulated in the Figures 1-6. $\max \left(\left|\boldsymbol{E}_{n}\right|\right)$ is calculated as the $99.9^{\text {th }}$ percentile of the absolute value of the normal component in the middle of the grey matter sheet. The stimulated area is the area in [\%] where the absolute value of the normal component of the field is greater or equal than $80 \%$ the maximum value. The RDM is a relative distance measure between the absolute value of the normal components of the fields in the in- and anti-phase conditions and indicates the similarity of the field 


\section{ACCEPTED MANUSCRIPT}

distributions generated by both conditions, irrespective of the temporal phase of the local electric fields. DotP measures the orthogonality of the normal component of the in- and anti-phase fields.

For the ds-tACS results to be more interpretable, we want the fields in the in- and antiphase conditions to have similar strengths, similar stimulated areas, to affect the same regions (low $\mathrm{RDM}$ ), and to be orthogonal to each other (DotP close to zero). From the Table, we can argue that the best solution explored here is the 2 rings montage with 4 channels (Figure 5 A-D). 


\section{References}

Alam, M., Truong, D.Q., Khadka, N., Bikson, M., 2016. Spatial and polarity precision of concentric high-definition transcranial direct current stimulation (HD-tDCS). Phys. Med. Biol. 61, 45064521. doi:10.1088/0031-9155/61/12/4506

Bächinger, M., Zerbi, V., Moisa, M., Polania, R., Liu, Q., Mantini, D., Ruff, C., Wenderoth, N., 2017. Concurrent tACS-fMRI Reveals Causal Influence of Power Synchronized Neural Activity on Resting State fMRI Connectivity. J. Neurosci. 37.

Bortoletto, M., Rodella, C., Salvador, R., Miranda, P.C., Miniussi, C., 2016. Reduced Current Spread by Concentric Electrodes in Transcranial Electrical Stimulation (tES). Brain Stimul. 9, 525-528. doi:10.1016/j.brs.2016.03.001

Bungert, A., Antunes, A., Espenhahn, S., Thielscher, A., 2016. Where does TMS stimulate the motor cortex? Combining electrophysiological measurements and realistic field estimates to reveal the affected cortex position. Cereb. Cortex to appear, 1-12. doi:10.1093/cercor/bhw292

Buzsaki, G., Draguhn, A., 2004. Neuronal Oscillations in Cortical Networks. Science (80-. ). 304, $1-4$.

Canolty, R.T., Knight, R.T., 2010. The functional role of cross-frequency coupling. Trends Cogn. Sci. 14, 506-15. doi:10.1016/j.tics.2010.09.001

Dale, a M., Fischl, B., Sereno, M.I., 1999. Cortical surface-based analysis. I. Segmentation and surface reconstruction. Neuroimage 9, 179-194. doi:10.1006/nimg.1998.0395

Dmochowski, J., Datta, A., Bikson, M., Su, Y., Parra, L., 2011. Optimized multi-electrode stimulation increases focality and intensity at target. J. Neural Eng. 8, 46011.

Fries, P., 2005. A mechanism for cognitive dynamics: Neuronal communication through neuronal coherence. Trends Cogn. Sci. doi:10.1016/j.tics.2005.08.011

Geuzaine, C., 2007. GetDP: a general finite-element solver for the de Rham complex. Proc. Appl. Math. Mech. 7, 4070029-4070030. doi:10.1002/pamm.2007 
Helfrich, R.F., Knepper, H., Nolte, G., Str??ber, D., Rach, S., Herrmann, C.S., Schneider, T.R., Engel, A.K., 2014. Selective Modulation of Interhemispheric Functional Connectivity by HDtACS Shapes Perception. PLoS Biol. 12. doi:10.1371/journal.pbio.1002031

Homan, R.W., Herman, J., Purdy, P., 1987. Cerebral location of international 10-20 system electrode placement. Electroencephalogr. Clin. Neurophysiol. 66, 376-382. doi:10.1016/00134694(87)90206-9

Huang, Y., Dmochowski, J.P., Su, Y., Datta, A., Rorden, C., Parra, L.C., 2013. Automated MRI segmentation for individualized modeling of current flow in the human head. J Neural Eng. 10.

Meijs, J.W.H., Weier, O.W., Peters, M.J., Van Oosterom, A., 1989. On the numerical accuracy of the boundary element method (EEG application). IEEE Trans. Biomed. Eng. 36, 1038-1049. doi:10.1109/10.40805

Miranda, P.C., Mekonnen, A., Salvador, R., Basser, P.J., 2014. Predicting the electric field distribution in the brain for the treatment of glioblastoma. Phys. Med. Biol. 59, 4137-4147. doi:10.1088/0031-9155/59/15/4137

Opitz, A., Falchier, A., Yan, C.-G., Yeagle, E.M., Linn, G.S., Megevand, P., Thielscher, A., Deborah A., R., Milham, M.P., Mehta, A.D., Schroeder, C.E., 2016. Spatiotemporal structure of intracranial electric fields induced by transcranial electric stimulation in humans and nonhuman primates. Sci. Rep. 6, 31236. doi:10.1038/srep31236

Opitz, a, Windhoff, M., Heidemann, R.M., Turner, R., Thielscher, a, 2011. How the brain tissue shapes the electric field induced by transcranial magnetic stimulation. Neuroimage $58,849-$ 859. doi:10.1016/j.neuroimage.2011.06.069

Polanía, R., Moisa, M., Opitz, A., Grueschow, M., Ruff, C.C., 2015. The precision of value-based choices depends causally on fronto-parietal phase coupling. Nat. Commun. 6, 8090. doi:10.1038/ncomms9090

Polanía, R., Nitsche, M.A., Korman, C., Batsikadze, G., Paulus, W., 2012. The importance of timing in segregated theta phase-coupling for cognitive performance. Curr. Biol. 22, 1314 
1318. doi:10.1016/j.cub.2012.05.021

Rahman, A., Reato, D., Arlotti, M., Gasca, F., Datta, D., Parra, L.C., Bikson, M., 2013. Cellular effects of acute direct current stimulation: somatic and synaptic terminal effects. J Physiol 591The, 2563-2578.

Saturnino, G.B., Antunes, A., Thielscher, A., 2015. On the importance of electrode parameters for shaping electric field patterns generated by tDCS. Neuroimage 120, 25-35. doi:10.1016/j.neuroimage.2015.06.067

Strüber, D., Rach, S., Trautmann-Lengsfeld, S.A., Engel, A.K., Herrmann, C.S., 2014. Antiphasic $40 \mathrm{~Hz}$ oscillatory current stimulation affects bistable motion perception. Brain Topogr. 27, 158-171. doi:10.1007/s10548-013-0294-х

Thielscher, A., Antunes, A., Saturnino, G.B., 2015. Field modeling for transcranial magnetic stimulation: A useful tool to understand the physiological effects of TMS? Proc. Annu. Int. Conf. IEEE Eng. Med. Biol. Soc. EMBS 2015-Novem, 222-225.

doi:10.1109/EMBC.2015.7318340

Violante, I.R., Li, L.M., Carmichael, D.W., Lorenz, R., Leech, R., Hampshire, A., Rothwell, J.C., Sharp, D.J., Fowler, J., Mackay, C., Moeller, S., Xu, J., Yacoub, E., Baselli, G., Ugurbil, K., Miller, K., Smith, S., 2017. Externally induced frontoparietal synchronization modulates network dynamics and enhances working memory performance. Elife 6, 91-95. doi:10.7554/eLife.22001

Windhoff, M., Opitz, A., Thielscher, A., 2013. Electric field calculations in brain stimulation based on finite elements: An optimized processing pipeline for the generation and usage of accurate individual head models. Hum. Brain Mapp. 34, 923-935. doi:10.1002/hbm.21479 
A
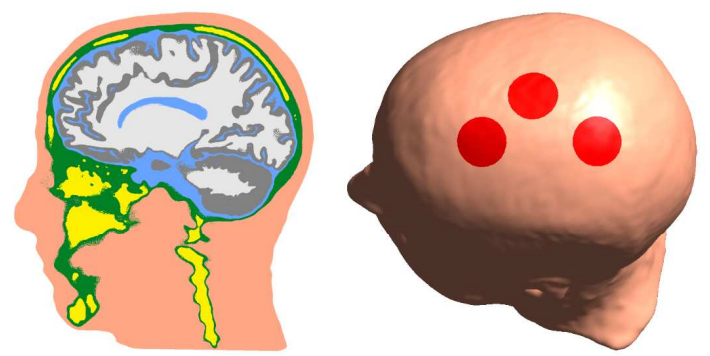

B

In-Phase

Anti-phase
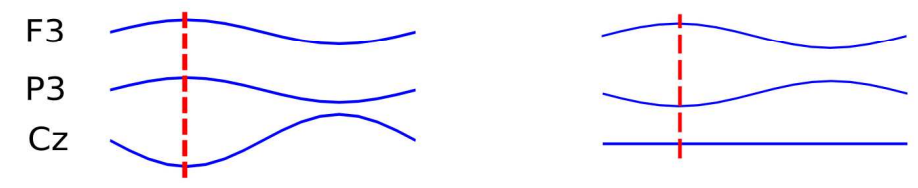

है

C
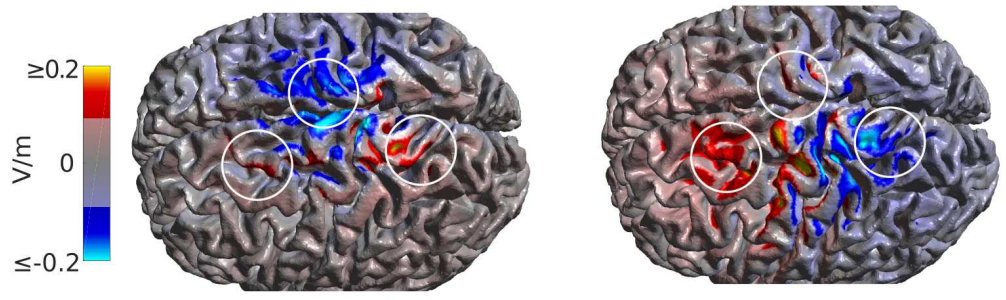

D
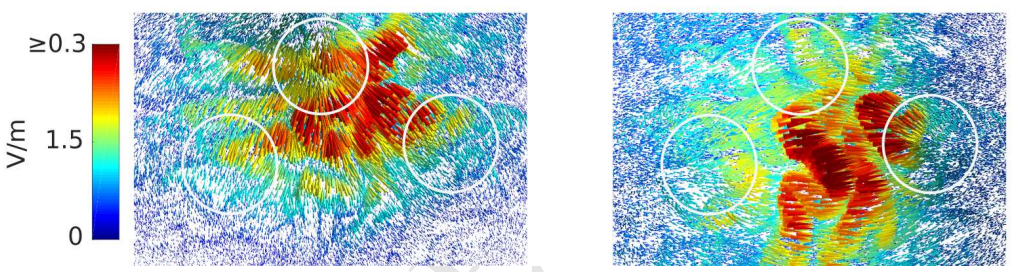
A

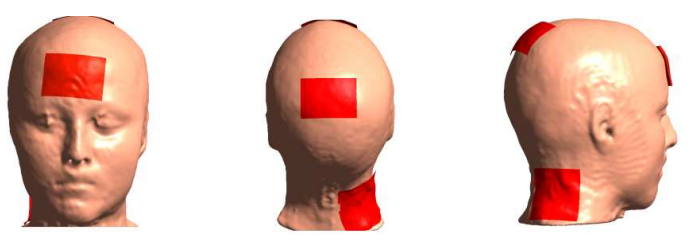

B

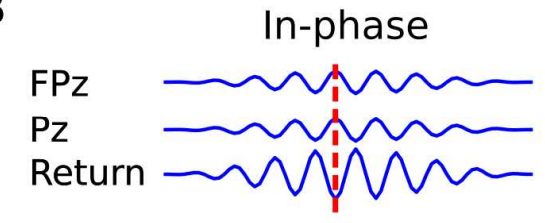

C
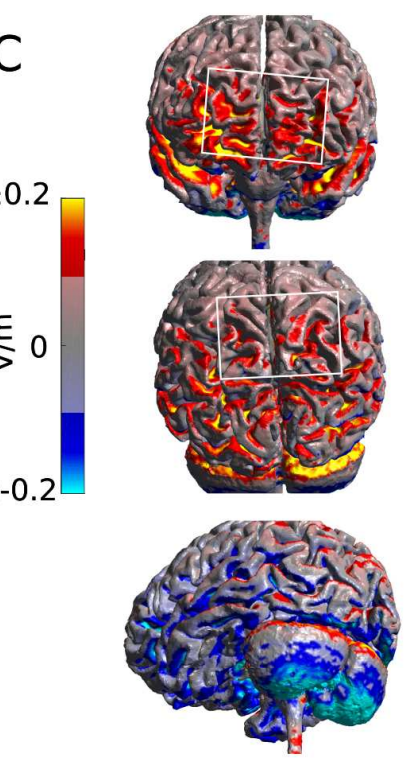

D
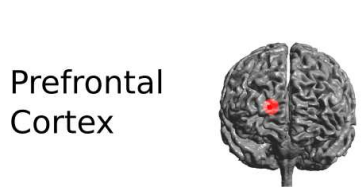

$$
\text { In-phase }
$$

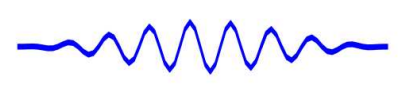

Posterior

Parietal

Cortex
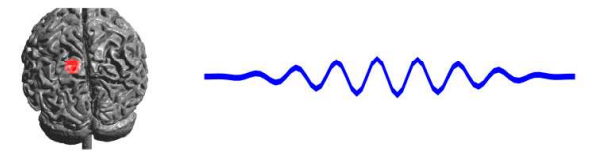

Cerebellum

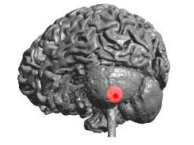

mMn

Motor

Cortex

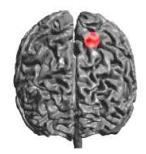

Orbitofrontal

Cortex

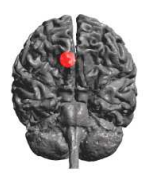

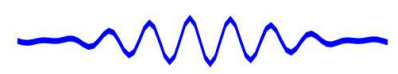
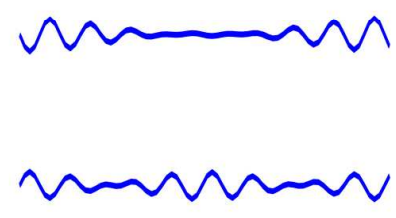

Anti-phase
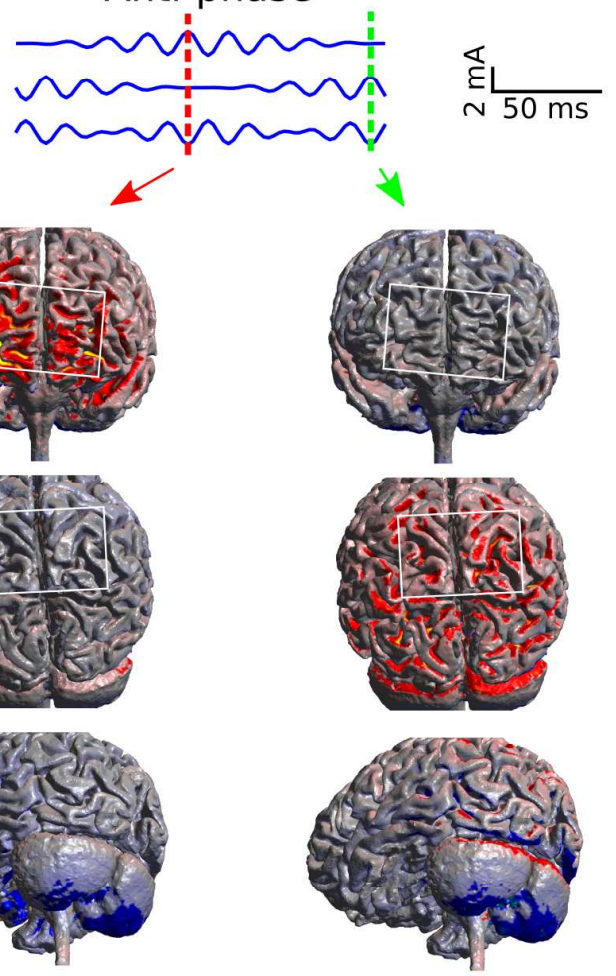

Anti-phase

$m$

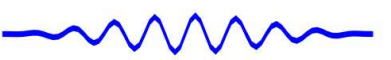

w w

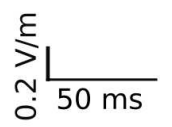


A

B
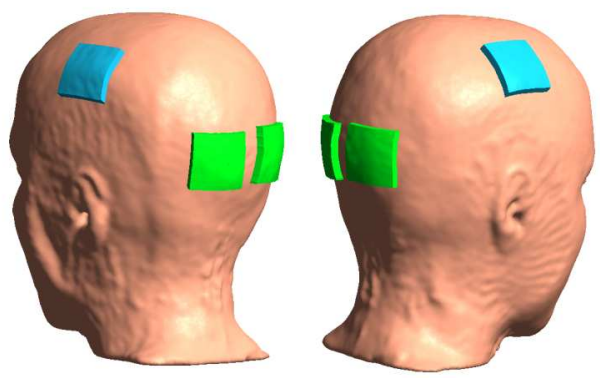

In-phase

Channel 1

Channel 2

$\stackrel{\text { हL }}{\rightarrow 10 \mathrm{~ms}}$

C

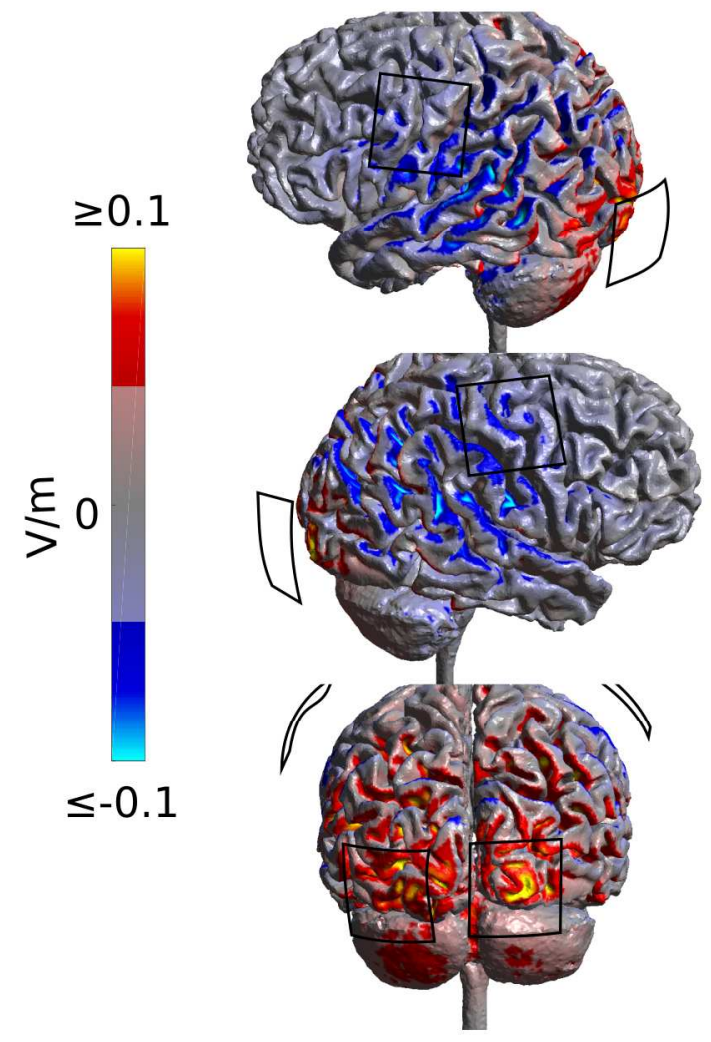

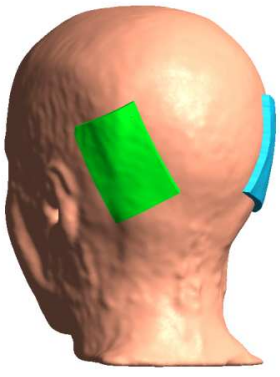

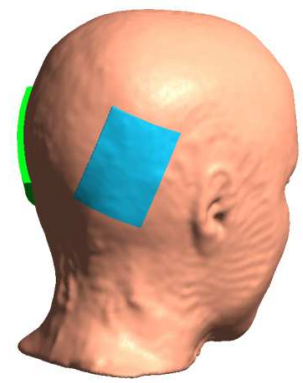

Anti-phase
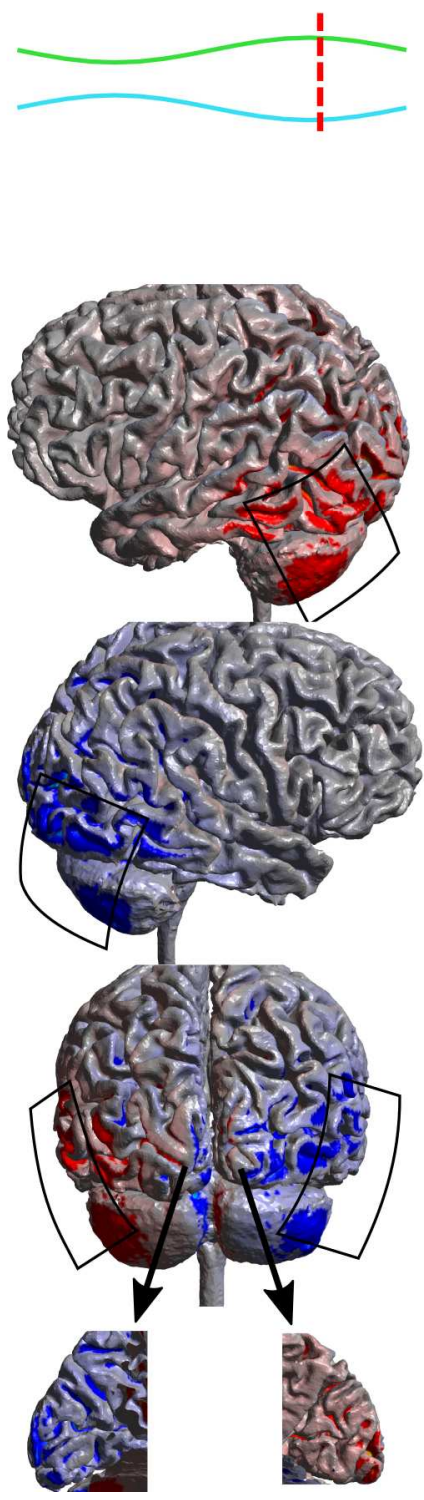
A

B

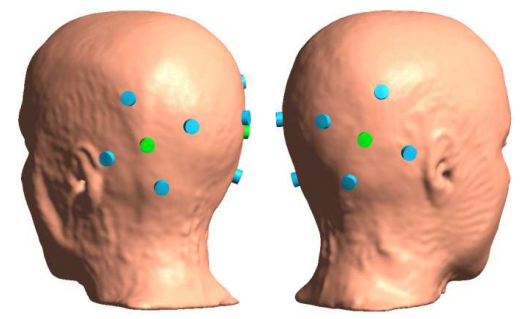

In-phase

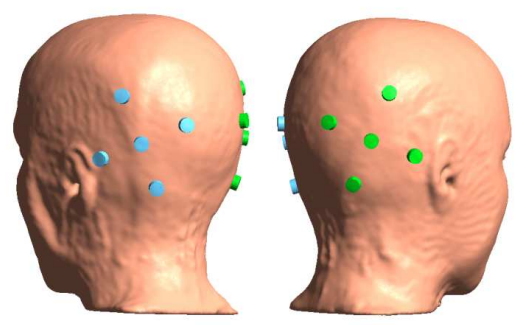

Anti-phase

Channel 1

Channel 2

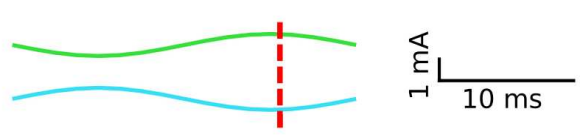

C
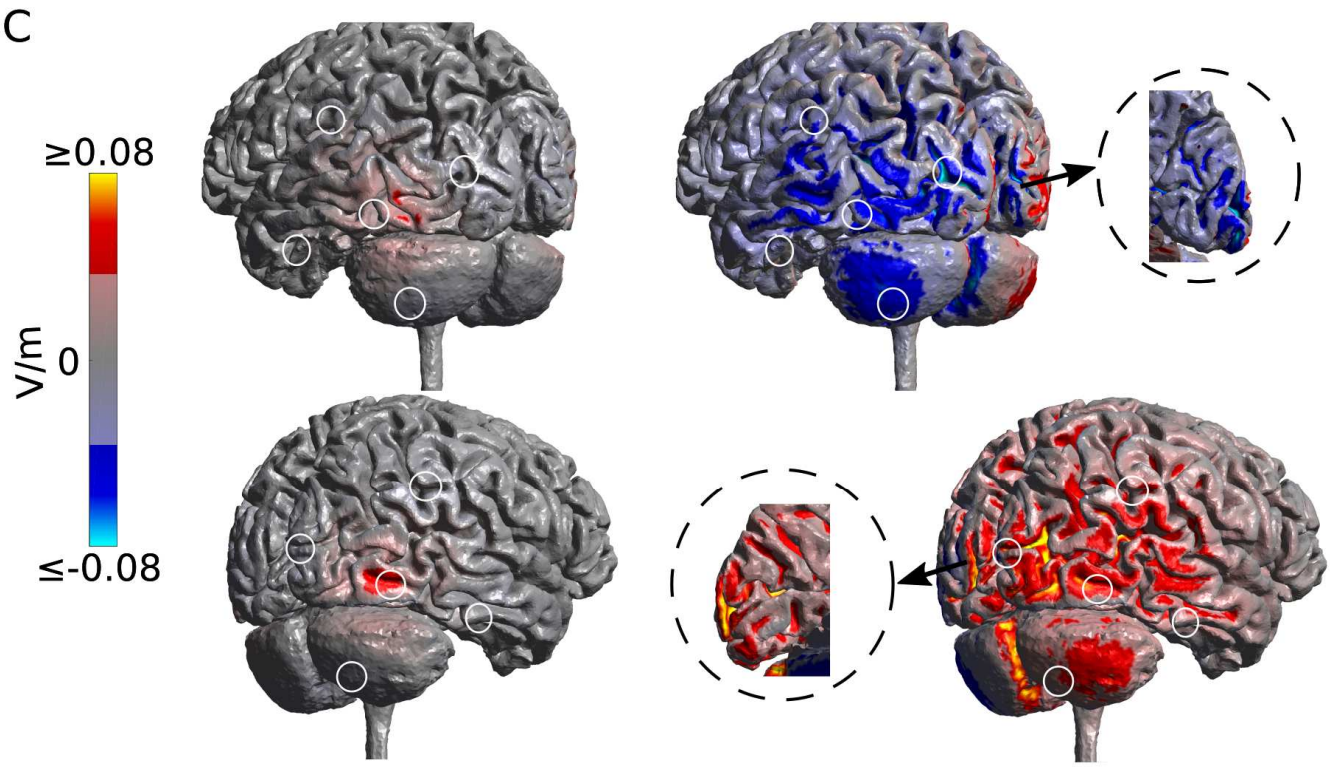
A
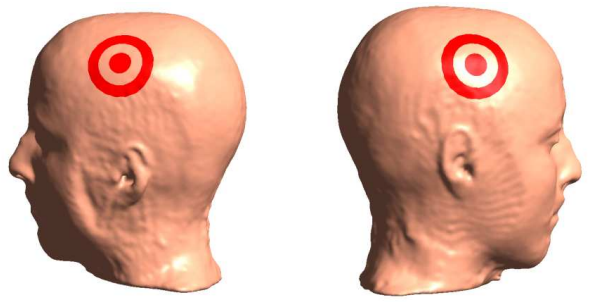

B

In-Phase

Anti-phase

C

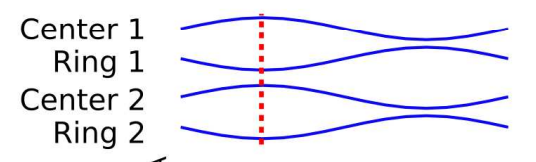
$\stackrel{\mathbb{\varepsilon}}{-1}$
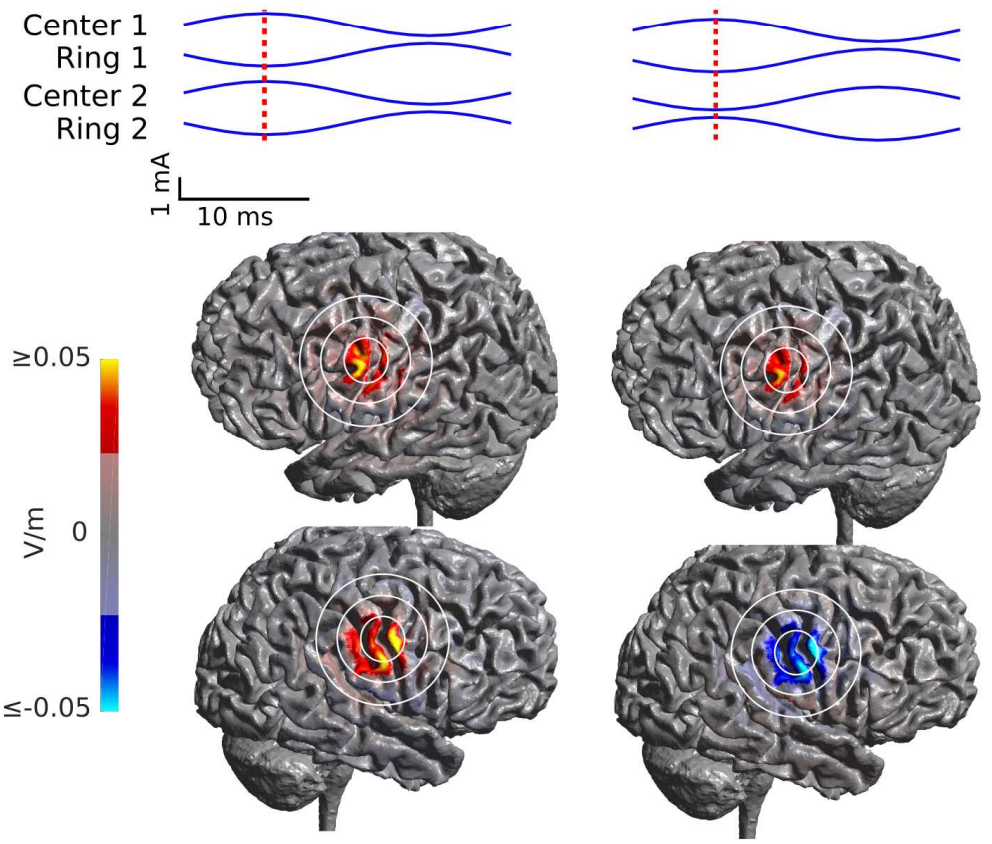

D
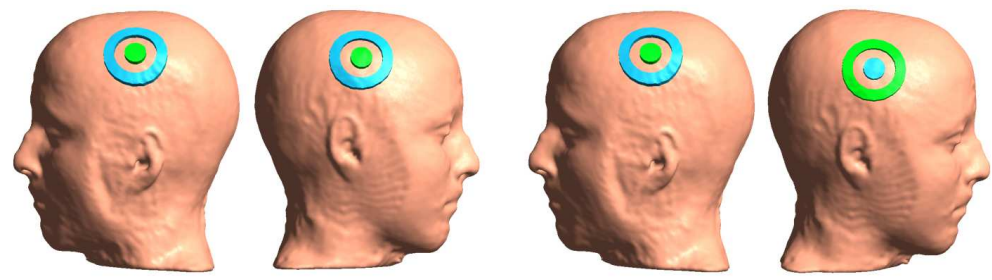

E

In-Phase

Anti-phase
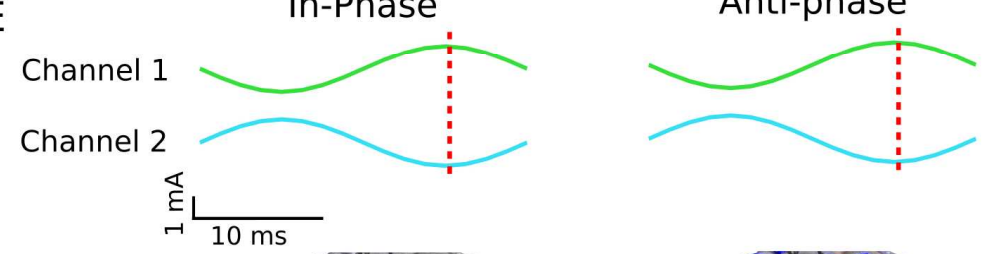

F
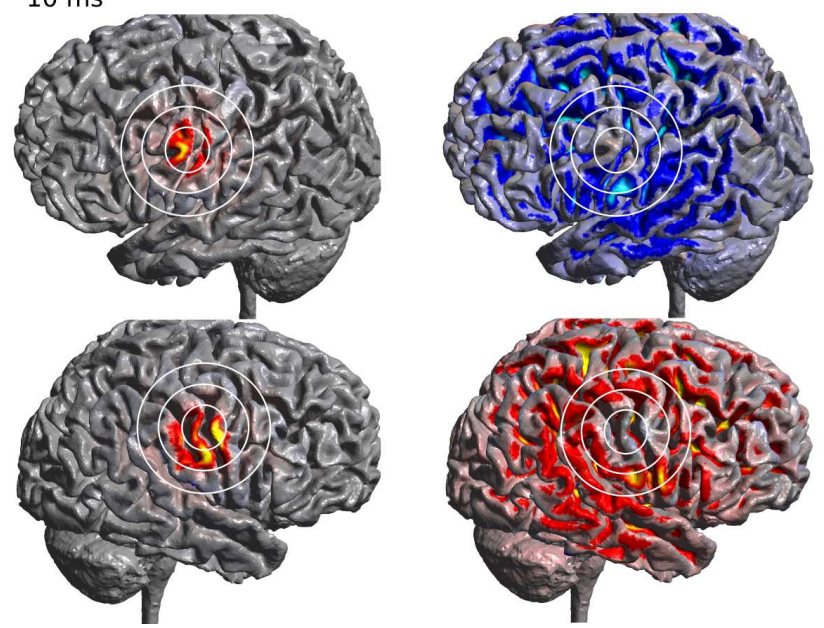
A

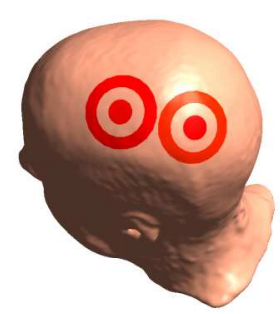

B

In-Phase

Center 1 Ring 1

Center 2

Ring 2

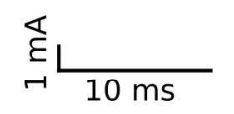

C

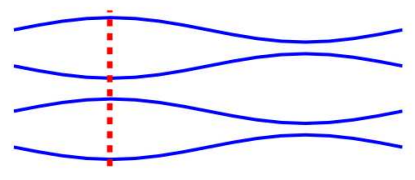

Anti-phase

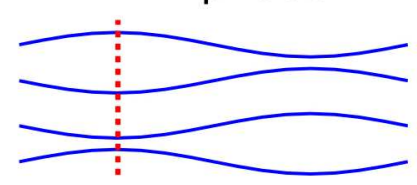

$\geq 0.04$

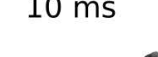

$$
\stackrel{\xi}{>} 0
$$

D

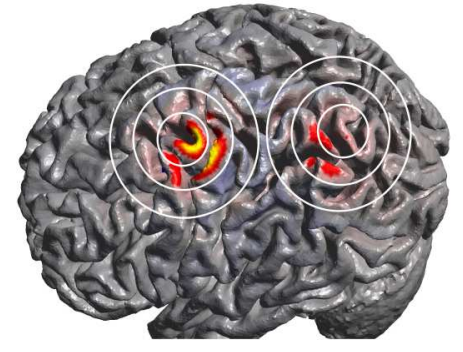

$\geq 0.06$
$\sum_{0} 0.03$
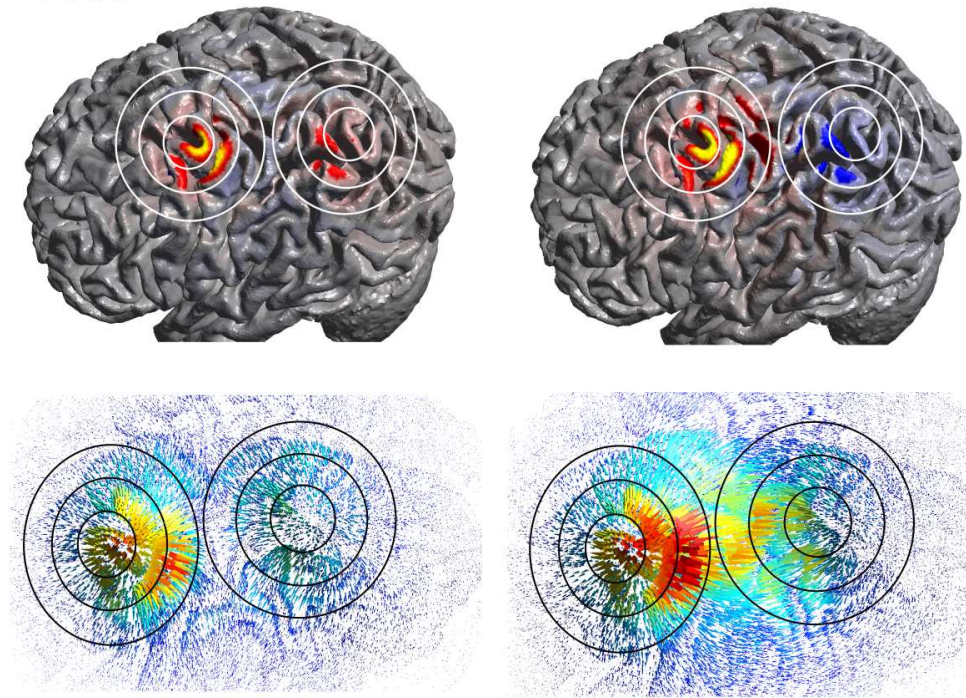

E
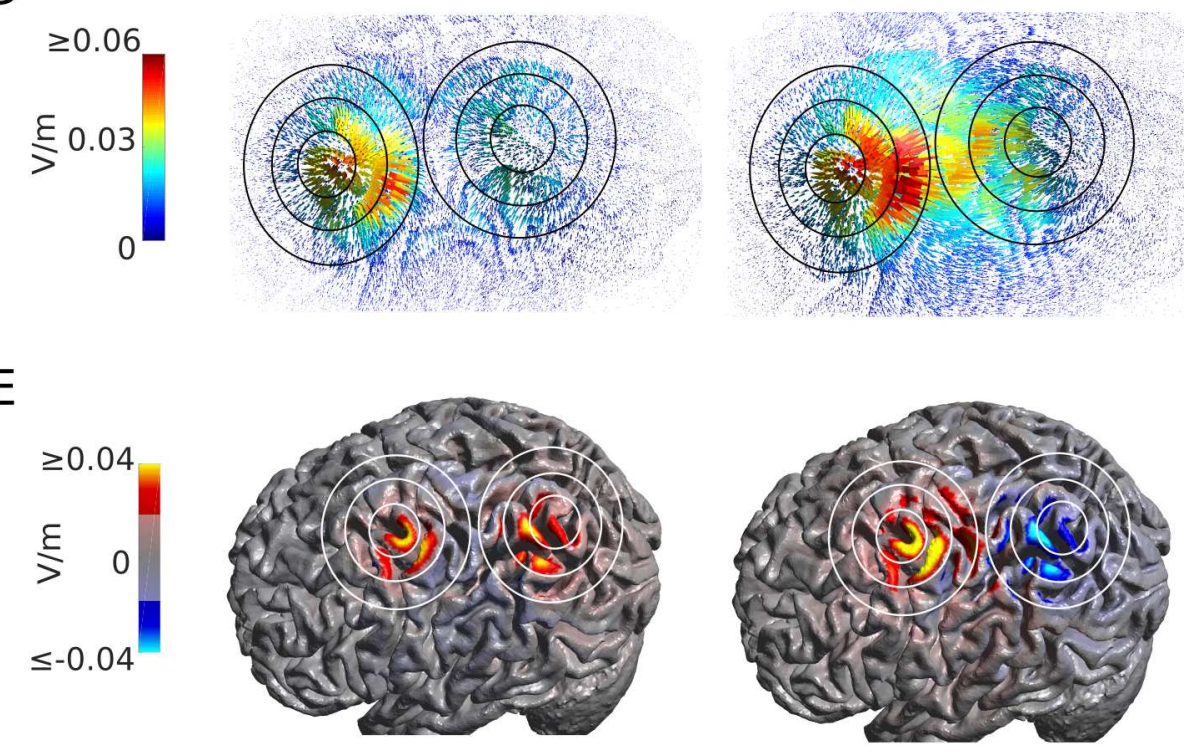

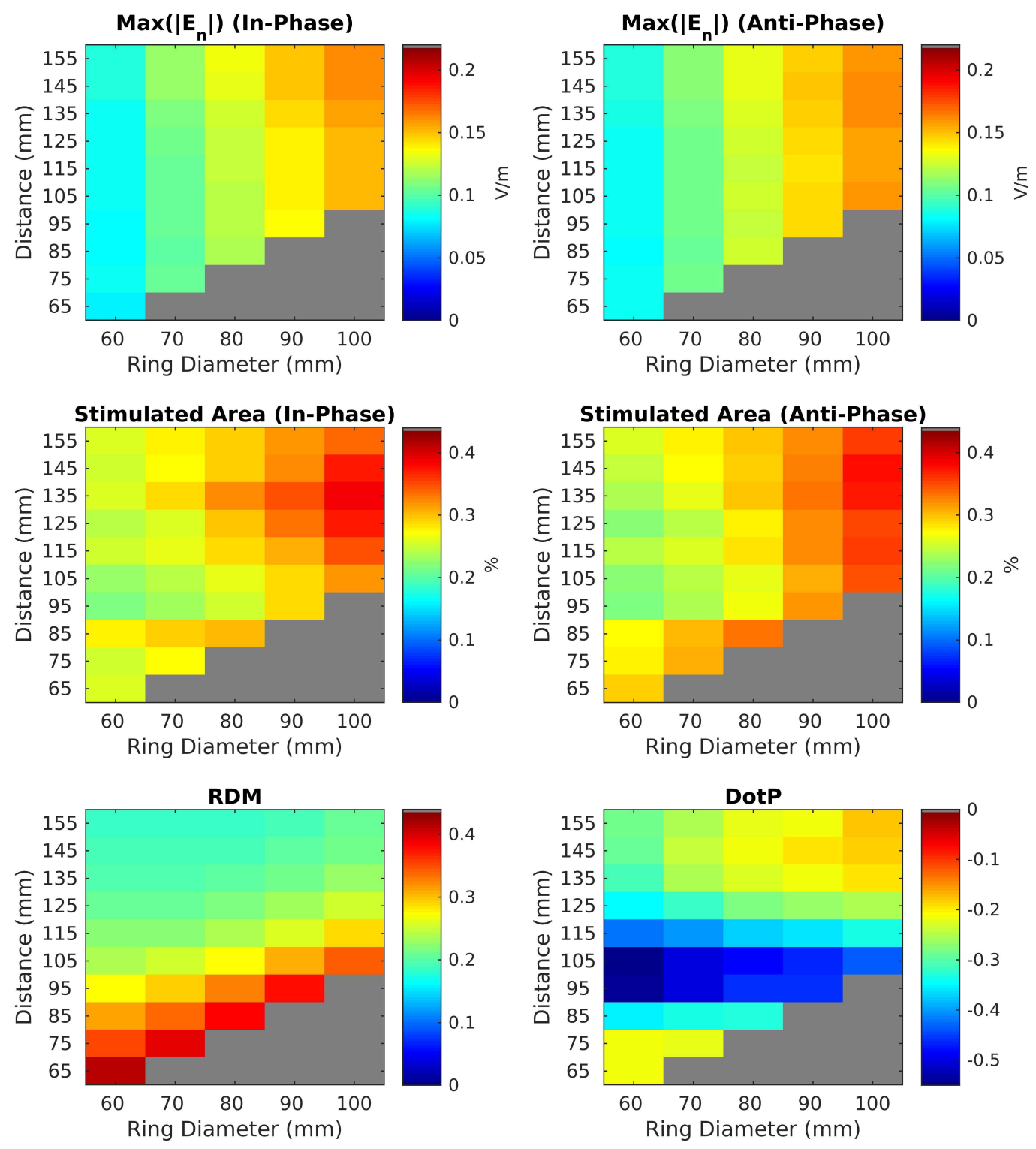